TRANSACTIONS OF THE AMERICAN MATHEMATICAL SOCIETY

Volume 348 , Number 11, November 1996

\title{
THE LYAPUNOV SPECTRUM OF FAMILIES OF TIME-VARYING MATRICES
}

\author{
FRITZ COLONIUS AND WOLFGANG KLIEMANN
}

\begin{abstract}
For $L^{\infty}$-families of time varying matrices centered at an unperturbed matrix, the Lyapunov spectrum contains the Floquet spectrum obtained by considering periodically varying piecewise constant matrices. On the other hand, it is contained in the Morse spectrum of an associated flow on a vector bundle. A closer analysis of the Floquet spectrum based on geometric control theory in projective space and, in particular, on control sets, is performed. Introducing a real parameter $\rho \geq 0$, which indicates the size of the $L^{\infty}$-perturbation, we study when the Floquet spectrum, the Morse spectrum, and hence the Lyapunov spectrum all coincide. This holds, if an inner pair condition is satisfied, for all up to at most countably many $\rho$-values.
\end{abstract}

\section{InTRODUCTION}

The real spectrum of a matrix $A \in g \ell(d, \mathbb{R})(=$ set of $d \times d$ matrices with real entries) can be characterized in two different ways: Via the exponential growth behavior of the solutions of $\dot{x}=A x$, i.e. via the real parts of eigenvalues, or topologically via the chain recurrent components of the induced equation on the projective space $\mathbb{P}^{d-1}$, i.e. via the (generalized) eigenspaces of eigenvalues with the same real part. The characterization using exponential growth rates was generalized by A.M. Lyapunov in his 1892 thesis [Ly] to time varying matrix functions $A(t)$ by introducing the concept, which is called now 'Lyapunov exponent': $\lambda^{ \pm}\left(x_{0}\right)=\limsup _{t \rightarrow \pm \infty} \frac{1}{t} \log \left|\varphi\left(t, x_{0}\right)\right|$, where $\varphi\left(\cdot, x_{0}\right)$ denotes the solution of $\dot{x}=A(t) x$ with $\varphi\left(0, x_{0}\right)=x_{0} \in \mathbb{R}^{d}$. Lyapunov identified the so-called 'regular matrix functions', which admit a decomposition of $\mathbb{R}^{d}$ into a direct sum of subspaces, in which the Lyapunov exponents are actually limits and independent of the time direction. For regular matrix functions, the stability behavior under small perturbations is characterized by the sign of the Lyapunov exponents, see e.g. [Ly], [Ha], [Ce], [BVGN].

For families of (time varying) matrix functions $\mathcal{A} \subset\{A: \mathbb{R} \rightarrow g \ell(d, \mathbb{R})\}$ the characterization of the entire set of Lyapunov exponents remains a problem, mainly for two reasons: The exponents may not depend continuously on $A$ (if $\mathcal{A}$ is endowed

Received by the editors January 25, 1994 and, in revised form, March 31, 1995.

1991 Mathematics Subject Classification. Primary 34D08, 93B05, 58F25.

Key words and phrases. Lyapunov exponents, Floquet exponents, Lyapunov regularity, Morse spectrum, control sets, chain control sets, chain recurrence.

This research was partially supported by DFG grant no. Co 124/8-2 and ONR grant no. N00014-93-1-0868. 
with some topology), and $\mathcal{A}$ may contain non-regular functions, for which the relation of the growth rates to those of the regular elements in $\mathcal{A}$ is not known in general.

Important progress can be made if one considers the time varying behavior as a flow on the set $\mathcal{A}$ (compare e.g. [Mi], [Sei], and [Sel] for early ideas in this direction.) Complementary results were obtained from this point of view in particular by Sacker/Sell, and by Oseledeč. Under the assumption that the flow on $\mathcal{A}$ has an invariant probability measure $\mu$, Oseledeč [Os] proved that with $\mu$-probability 1 the matrix functions are regular. This measure-theoretic result, in which the set of regular functions can be topologically thin (see e.g. [Ma], p. 264), describes subsets of the entire Lyapunov spectrum of $\mathcal{A}$ as regular exponents, i.e. it represents an approximation of the entire spectrum 'from the inside'. In contrast the topological approach of Sacker and Sell constructs via (uniform) exponential splitting in $\mathcal{A} \times \mathbb{R}^{d}$ the dichotomy spectrum of $\mathcal{A}$ as those $\lambda \in \mathbb{R}$ for which the flow, multiplied by $e^{\lambda t}$, has no exponential dichotomy, see [SaS]. The dichotomy (or 'dynamical') spectrum contains the Lyapunov spectrum, and its extreme points are actually Lyapunov exponents, see [JPS]. Both results play an important role in the theory of dynamical systems, and recently also for stochastic dynamics and nonlinear control theory.

All approaches mentioned so far start from the analysis of exponential growth rates for a family $\mathcal{A}$ of time-varying matrix functions with a flow on $\mathcal{A}$. According to the situation for constant matrices, one can also try to start with a generalization of the 'eigenspaces' of $\mathcal{A}$, projected onto the projective space $\mathbb{P}^{d-1}$, i.e. with topological properties of the induced flow on $\mathcal{A} \times \mathbb{P}^{d-1}$, and construct suitable spectra over these sets. This is, for $L^{\infty}$-families $\mathcal{A}$ centered at a constant matrix $A_{0} \in g \ell(d, \mathbb{R})$, the approach of this paper. We construct the Morse spectrum over the finest Morse decomposition of the projected flow as an 'outer approximation' of the Lyapunov spectrum. These results, for the more general situation of linear flows on vector bundles, appear in $[\mathrm{CKj}]$. They are briefly reviewed here for our special case in Section 3. An 'inner approximation' is obtained by constructing the 'Floquet spectrum' over the projected eigenspaces of piecewise constant, periodic matrix functions in $\mathcal{A}$, see Section 4. This is related to the action of a Lie semigroup (the systems semi-group) on projective space. It turns out that for a point $(A, x) \in \mathcal{A} \times \mathbb{R}^{d} \backslash\{0\}$ the Lyapunov exponent $\lambda(A, x)$ is in the closure of the Floquet spectrum, if the $\omega$-limit set of $(A, x)$ on $\mathbb{P}^{d-1}$ intersects the interior of the set of projected eigenspaces of piecewise constant, periodic functions in $\mathcal{A}$.

Hence the gap between these two spectra can be analyzed by 'closing' the chains of the Morse sets in the interior of the sets of projected eigenspaces. To this end, we introduce in Section 5 a real parameter $\rho \geq 0$, indicating the size of the $L^{\infty}$ perturbation range. It is shown that under an inner pair condition (which is necessary and sufficient for the existence of a suitable embedding of the Morse sets, see $[\mathrm{CKh}])$ there are at most countably many $\rho$-values for which the spectra can differ. For all other perturbation ranges we obtain that the closure of the Floquet spectrum, the Lyapunov spectrum, and the Morse spectrum agree.

This paper is based on a variety of results and methods from the theory of dynamical systems and nonlinear geometric control theory. It presents a synthesis of the ideas either developed or put into action in our previous papers [CKa], [CKc], $[\mathrm{CKe}],[\mathrm{CKf}],[\mathrm{CKg}],[\mathrm{CKh}],[\mathrm{CKj}]$, which mainly originated from our efforts to understand the spectral properties of families of time varying matrices. A number 
of applications and related problems in control theory and in stochastic dynamics are discussed in $[\mathrm{CKb}],[\mathrm{CKd}]$, [CKi] and $[\mathrm{AKO}],[\mathrm{AK}]$.

\section{Problem Formulation}

Consider the $L^{\infty}$-family of time varying $d \times d$ matrices

$$
A_{0}+\sum_{i=1}^{m} u_{i}(t) A_{i}=: A(u(t))
$$

where $A_{j} \in g \ell(d, \mathbb{R}), j=0 \ldots m$, are given and the perturbation $\left(u_{i}(t)\right)_{i=1 \ldots m}=$ : $u(t)$ is of the form

$$
u \in \mathcal{U}:=\{u: \mathbb{R} \rightarrow U ; \text { measurable }\}
$$

with $U \subset \mathbb{R}^{m}$ compact and convex with $0 \in$ int $U$.

We are interested in a characterization of the entire Lyapunov spectrum of (2.1), i.e. in all Lyapunov exponents

$$
\lambda(u, x):=\limsup _{t \rightarrow \infty} \frac{1}{t} \log |\varphi(t, x, u)|,
$$

where $\varphi(t, x, u)$ solves the linear differential equation

$$
\dot{x}=\left(A_{0}+\sum_{i=1}^{m} u_{i}(t) A_{i}\right) x \text { in } \mathbb{R}^{d}
$$

with $\varphi(0, x, u)=x \neq 0$. We define the Lyapunov spectrum of (2.1) as

$$
\Sigma_{L y}=\left\{\lambda(u, x) ;(u, x) \in \mathcal{U} \times \mathbb{R}^{d}, x \neq 0\right\} .
$$

The Lyapunov exponents of (2.1) define a map

$$
\lambda: \mathcal{U} \times \mathbb{R}^{d} \backslash\{0\} \rightarrow \mathbb{R}
$$

in fact, they are defined on a flow associated with the time varying differential equations (2.4), which is given by

$$
\begin{aligned}
& \Phi: \mathbb{R} \times \mathcal{U} \times \mathbb{R}^{d} \longrightarrow \mathcal{U} \times \mathbb{R}^{d}, \\
& \Phi_{t}(u, x)=\left(\theta_{t} u, \varphi(t, x, u)\right)
\end{aligned}
$$

where $\theta_{t} u(\cdot):=u(t+\cdot)$ is the usual shift by $t$. If one endows $\mathcal{U}$ with the weak* topology of $L^{\infty}\left(\mathbb{R}, \mathbb{R}^{m}\right)=L^{1}\left(\mathbb{R}, \mathbb{R}^{m}\right)^{*}$, then $\Phi$ becomes a continuous flow, see [CKf] for details. The flow point of view allows us to use concepts and techniques from topological dynamics for the analysis of the Lyapunov spectrum.

The exponential growth behavior of linear differential equations (or linear flows) can be studied via the associated (angular) system on the projective space $\mathbb{P}^{d-1}$ : The angular component of (2.4) is

$$
\dot{s}=h_{0}(s)+\sum_{i=1}^{m} u_{i}(t) h_{i}(s)=: h(u, s) \text { on } \mathbb{P}^{d-1}
$$

where $h_{j}(s)=\left(A_{j}-s^{T} A_{j} s\right) s$ for $j=0 \ldots m$. Solving the corresponding equation for the radial component yields for the Lyapunov exponent

$$
\lambda(u, x)=\limsup _{t \rightarrow \infty} \frac{1}{t} \int_{0}^{t} q\left(u(\tau), s\left(\tau, s_{0}, u\right)\right) d \tau
$$


with $q(u, s)=q_{0}(s)+\sum_{i=1}^{m} u_{i}(t) q_{i}(s), q_{j}(s)=s^{T} A_{j} s$, and $s_{0}=\frac{x}{|x|}$, the projection of $x \in \mathbb{R}^{d} \backslash\{0\}$ onto $\mathbb{P}^{d-1}$. Here $s\left(\tau, s_{0}, u\right)$ denotes the solution of (2.8) with $s\left(0, s_{0}, u\right)=s_{0} \in \mathbb{P}^{d-1}$. With the family of (nonlinear) differential equations (2.8) we associate the projected flow

$$
\begin{aligned}
& \mathbb{P} \Phi: \mathbb{R} \times \mathcal{U} \times \mathbb{P}^{d-1} \longrightarrow \mathcal{U} \times \mathbb{P}^{d-1} \\
& \mathbb{P} \Phi_{t}\left(u, s_{0}\right)=\left(\theta_{t} u, s\left(t, s_{0}, u\right)\right) .
\end{aligned}
$$

Hence the Lyapunov exponents of (2.1) are defined over $\mathbb{P} \Phi$ as a map

$$
\lambda: \mathcal{U} \times \mathbb{P}^{d-1} \longrightarrow \mathbb{R},
$$

and the Lyapunov spectrum (2.5) of (2.1) can be written as

$$
\Sigma_{L y}=\left\{\lambda(u, x) ;(u, x) \in \mathcal{U} \times \mathbb{P}^{d-1}\right\} .
$$

Our goal is to characterize $\Sigma_{L y}$. This problem is difficult, mainly for two reasons:

- The map $\lambda$ in (2.11) need not be continuous nor semi-continuous, compare e.g. Vinograd's example ([Ha], p. 325).

- For all perturbation ranges $U$ as above, the set of admissible perturbations $\mathcal{U}$ contains functions $u$ such that $A_{0}+\Sigma u_{i}(t) A_{i}$ is not Lyapunov regular, i.e. the lim sup's in (2.3) or (2.9) need not be limits and a regular basis and associated Lyapunov spaces need not exist, compare e.g. Cesari [Ce], Hahn [Ha].

In the following sections we will use properties of the flow $\mathbb{P} \Phi$ in (2.10) to characterize $\Sigma_{L y}$ for 'almost all' perturbation ranges. The 'outer' approximation uses chain-recurrent components of $\mathbb{P} \Phi$ and the associated Morse spectrum, while the 'inner' approximation considers eigenspaces of periodic perturbations and associated Floquet exponents. Ideas from geometric nonlinear control theory are used to combine the two approaches yielding the main result of the paper.

\section{The Morse Spectrum}

In this section we present a brief review of some results from [CKj] that allow us to approximate the Lyapunov spectrum (2.5) from the 'outside' by the Morse spectrum. Compare also $[\mathrm{Co}]$ for some basic concepts.

For $\varepsilon, T>0$ an $(\varepsilon, T)$-chain $\zeta$ of $\mathbb{P} \Phi$ is given by $n \in \mathbb{N}, T_{0}, \ldots, T_{n-1} \geq T$, and $\left(u_{0}, p_{0}\right), \ldots,\left(u_{n}, p_{n}\right)$ in $\mathcal{U} \times \mathbb{P}^{d-1}$ with $d\left(\mathbb{P} \Phi\left(T_{i},\left(u_{i}, p_{i}\right)\right),\left(u_{i+1}, p_{i+1}\right)\right)<\varepsilon$ for $i=0,1, \ldots, n-1$. Here $d(\cdot, \cdot)$ denotes a metric on $\mathcal{U} \times \mathbb{P}^{d-1}$, see [CKf], Lemma 2.1. Denote the chain recurrent set of $\mathbb{P} \Phi$ by $\mathcal{R}$, i.e. $\mathcal{R}=\left\{(u, p) \in \mathcal{U} \times \mathbb{P}^{d-1}\right.$; for all $\varepsilon>0, T>0$ there exists an $(\varepsilon, T)$-chain with $\left.\left(u_{0}, p_{0}\right)=\left(u_{n}, p_{n}\right)=(u, p)\right\}$. The set $\mathcal{R}$ has $\ell$ connected components $\mathcal{E}_{1}, \ldots, \mathcal{E}_{\ell} \quad(1 \leq \ell \leq d)$, the Morse sets of $\mathbb{P} \Phi$. Their projections $E_{j}:=\pi_{\mathbb{P}} \mathcal{E}_{j} \subset \mathbb{P}^{d-1}$ are the chain control sets of (2.8), see [CKg], Theorem 4.9.

Define the finite time exponential growth rate of a chain $\zeta$ (or 'chain exponent') by

$$
\lambda(\zeta)=\left(\sum_{i=0}^{n-1} T_{i}\right)^{-1} \sum_{i=0}^{n-1}\left(\log \left|\varphi\left(T_{i}, x_{i}, u_{i}\right)\right|-\log \left|x_{i}\right|\right)
$$


where $\frac{x_{i}}{\left|x_{i}\right|}=p_{i} \in \mathbb{P}^{d-1}$. The Morse spectrum of $\Phi$ on $\mathcal{E}_{j}$ is given by

$$
\begin{aligned}
\Sigma_{M o}\left(\mathcal{E}_{j}, \Phi\right)= & \left\{\lambda \in \mathbb{R} ; \text { there are } \varepsilon^{k} \rightarrow 0, T^{k} \rightarrow \infty \text { and }\left(\varepsilon^{k}, T^{k}\right) \text {-chains } \zeta^{k}\right. \\
& \text { in } \left.\mathcal{E}_{j} \text { with } \lambda\left(\zeta^{k}\right) \rightarrow \lambda \text { as } k \rightarrow \infty\right\} ;
\end{aligned}
$$

we abbreviate this as $\Sigma_{M o}\left(E_{j}\right)$ and obtain the Morse spectrum of $\Phi$ as

$$
\Sigma_{M o}=\bigcup_{j=1}^{\ell} \Sigma_{M o}\left(E_{j}\right)
$$

(see [CKj], Definition 3.2).

We collect some basic facts on the Morse spectrum of $\Phi$ in the following theorem.

\subsection{Theorem.}

(i) For $j=1, \ldots, \ell, \Sigma_{M o}\left(E_{j}\right)$ is a compact interval, i.e. it has the form $\Sigma_{M o}\left(E_{j}\right)$ $=\left[\kappa^{*}\left(E_{j}\right), \kappa\left(E_{j}\right)\right]$.

(ii) $\Sigma_{L y} \subset \Sigma_{M o}$ and $\kappa^{*}\left(E_{j}\right), \kappa\left(E_{j}\right)$ are actually Lyapunov exponents for some $\left(u_{j}^{*}, p_{j}^{*}\right),\left(u_{j}, p_{j}\right) \in \mathcal{E}_{j}, j=1, \ldots, \ell$.

(iii) $\mathcal{U} \times \mathbb{R}^{d}=\bigoplus_{j=1}^{\ell} \mathcal{V}_{j}$ (Whitney sum), where $\mathcal{V}_{j}:=\left\{(u, x) \in \mathcal{U} \times \mathbb{R}^{d} ; x \neq 0\right.$ implies $\left.(u, \mathbb{P} x) \in \mathcal{E}_{j}\right\}, j=1, \ldots, \ell$.

Proof. (i) follows from [CKj], Theorem 3.6. Assertion (ii) follows from [CKj], Theorems 3.7 and 4.6. For assertion (iii) see [CKj], Theorem 3.1 and note that the base space $\mathcal{U}$ for $\Phi$ is chain recurrent by [CKf], Lemma 4.5 .

The following example shows that the intervals of the Morse spectrum need not be disjoint.

3.2. Example. Consider the following family of $2 \times 2$ matrices

$$
\left(\begin{array}{cc}
1 & \frac{3}{4} \\
\frac{3}{4} & 1
\end{array}\right)+u_{1}(t)\left(\begin{array}{ll}
1 & 0 \\
0 & 1
\end{array}\right)+u_{2}(t)\left(\begin{array}{ll}
0 & 1 \\
0 & 0
\end{array}\right)+u_{3}(t)\left(\begin{array}{ll}
0 & 0 \\
1 & 0
\end{array}\right)
$$

with $U=[-1,1] \times\left[-\frac{1}{4}, \frac{1}{4}\right] \times\left[-\frac{1}{4}, \frac{1}{4}\right]$. The chain control sets of the projected system on $\mathbb{P}^{1}$ in $\mathbb{R}^{2}$ are given by

$$
\begin{aligned}
& E_{1}=\pi_{\mathbb{P}}\left\{\left(\begin{array}{l}
x_{1} \\
x_{2}
\end{array}\right) \in \mathbb{R}^{2} ; x_{2}=\alpha x_{1}, \alpha \in[-\sqrt{2},-1 / \sqrt{2}]\right\}, \\
& E_{2}=\pi_{\mathbb{P}}\left\{\left(\begin{array}{l}
x_{1} \\
x_{2}
\end{array}\right) \in \mathbb{R}^{2} ; x_{2}=\alpha x_{1}, \alpha \in[1 / \sqrt{2}, \sqrt{2}]\right\}
\end{aligned}
$$

where $\pi_{\mathbb{P}}$ denotes the projection of $\mathbb{R}^{2}$ onto $\mathbb{P}^{1}$. The Lyapunov exponents of the family (3.3) for constant $u \in U$ (i.e. the real parts of the eigenvalues) form two intervals $I_{1}=\left[-1, \frac{3}{2}\right]$ and $I_{2}=\left[\frac{1}{2}, 3\right]$, i.e. $I_{j} \subset \Sigma_{M o}\left(E_{j}\right), j=1,2$. Hence $\left[\frac{1}{2}, \frac{3}{2}\right]=$ $I_{1} \cap I_{2} \subset \Sigma_{M o}\left(E_{1}\right) \cap \Sigma_{M o}\left(E_{2}\right)$.

(In fact, more is true in this 2-dimensional situation with two different chain control sets: By [Jo], Theorem 4.1 it follows that all possible Lyapunov exponents are contained in the intervals of real parts of eigenvalues. Hence we have $I_{1} \cup I_{2}=$ $\Sigma_{L y}=\Sigma_{M o}$ here.)

3.3. Remark. Since the chain control sets $E_{j}, j=1, \ldots, \ell$, are projections of the Morse sets of the flow $\mathbb{P} \Phi$, they can be ordered according to the order of Morse sets, see $[\mathrm{Co}]$. Note that in our context the flow $\theta$ on $\mathcal{U}$ is chain recurrent, hence 
we obtain from Corollary 4.10 in [CKj]: If $E_{i} \prec E_{j}$, then $\kappa^{*}\left(E_{i}\right)<\kappa^{*}\left(E_{j}\right)$, and $\kappa\left(E_{i}\right)<\kappa\left(E_{j}\right)$. Therefore, the minimum and the maximum of the intervals of the Morse spectrum are separated.

\section{The Floquet Spectrum}

An 'inner' approximation of the Lyapunov spectrum of the family (2.1) can be obtained by considering the Floquet exponents of the periodic matrix functions in (2.1). A priori it is not clear that this part of the Lyapunov spectrum forms intervals. Therefore, we impose a nondegeneracy condition that allows us to use geometric control theory for the proof of certain properties of the Floquet spectrum.

Throughout this section we assume

$$
\operatorname{dim} \mathcal{L} \mathcal{A}\{h(u, \cdot) ; u \in U\}(p)=d-1 \text { for all } p \in \mathbb{P}^{d-1} .
$$

Here $h(u, \cdot)$ denotes the vector field of the projected system on $\mathbb{P}^{d-1}$ as in (2.8) for constant $u \in U, \mathcal{L A}$ denotes the Lie algebra generated by these vector fields, and $\operatorname{dim} \mathcal{L} \mathcal{A}(p)$ is the dimension of the distribution generated by $\mathcal{L} \mathcal{A}$ in the tangent space $T_{p} \mathbb{P}^{d-1}$ of $\mathbb{P}^{d-1}$ at the point $p \in \mathbb{P}^{d-1}$. The assumption (H) is equivalent to the requirement that the system (2.8), interpreted as a (analytic) control system on $\mathbb{P}^{d-1}$, is locally accessible, see e.g. [Is].

We consider piecewise constant, periodic matrix functions of (2.1). Their fundamental matrices form the systems group $\mathcal{G}$ and semigroup $\mathcal{S}$, defined as

$$
\begin{aligned}
\mathcal{G}:= & \left\{\exp \left(t_{n} B_{n}\right) \cdots \exp \left(t_{1} B_{1}\right) ; t_{j} \in \mathbb{R}, B_{j}=A\left(u_{j}\right)\right. \\
& \left.\quad \text { for some } u_{j} \in U, j=1 \ldots n, n \in \mathbb{N}\right\} \\
\mathcal{S}:= & \left\{\exp \left(t_{n} B_{n}\right) \cdots \exp \left(t_{1} B_{1}\right) ; t_{j} \geq 0, B_{j}=A\left(u_{j}\right)\right. \\
& \left.\quad \text { for some } u_{j} \in U, j=1 \ldots n, n \in \mathbb{N}\right\} .
\end{aligned}
$$

Note that $\mathcal{G}$ is a Lie group acting naturally on $\mathbb{R}^{d} \backslash\{0\}$ and on $\mathbb{P}^{d-1}$. For $t>0$ denote by $\mathcal{S}_{\leq t}$ the subset of $\mathcal{S}$ with $\sum_{j=1}^{n} t_{j} \leq t$, then Assumption $(\mathrm{H})$ implies that the interior int $\mathcal{S}_{\leq t}$ of $\mathcal{S}_{\leq t}$ in $\mathcal{G}$ is non-empty. For $g \in G \ell(d, \mathbb{R})$ let spec $g$ be the spectrum of $g$, and $E(\lambda)$ the (generalized) eigenspace of $\lambda \in$ spec $g$. We introduce the notation

$$
\begin{aligned}
V & =\{E(\lambda) ; \lambda \in \operatorname{spec} g, g \in \text { int } \mathcal{S}\} \subset \mathbb{R}^{d}, \text { and } \\
\mathbb{P} V & =\{\mathbb{P} E(\lambda) ; \lambda \in \operatorname{spec} g, g \in \text { int } \mathcal{S}\} \subset \mathbb{P}^{d-1},
\end{aligned}
$$

where, for a set $A \subset \mathbb{R}^{d}, \mathbb{P} A$ is the projection of $A \backslash\{0\}$ onto $\mathbb{P}^{d-1}$. Recall that a main control set $D$ for the system $(2.8)$ is a maximal subset of $\mathbb{P}^{d-1}$ with nonvoid interior such that $D \subset c \ell(S . s)$ for all $s \in D$.

The following result was proved in [CKg], Theorem 3.10.

4.1. Theorem. Let assumption $(\mathrm{H})$ be satisfied.

(i) The connected components of $\mathbb{P} V$ are the interiors of the main control sets $D_{1} \ldots D_{k}$ of $(2.8)$ and $1 \leq k \leq d$.

(ii) The main control sets are linearly ordered by

$D_{i} \prec D_{j}$ if there exist $x_{i} \in D_{i}, x_{j} \in D_{j}, t>0$, and $g \in \mathcal{S}_{t}$ with $g x_{i}=x_{j}$.

We enumerate the control sets such that $D_{1} \prec D_{2} \prec \cdots \prec D_{k}$. 
(iii) For every $g \in$ int $\mathcal{S}_{\leq t}$ and every main control $D$ there exists $\mu \in$ spec $g$ with $\mathbb{P} E(\mu) \subset$ intD. Furthermore, if $\mu, \mu^{\prime} \in \operatorname{spec} g$ with $\operatorname{Re} \mu<R e \mu^{\prime}$, and $\mathbb{P} E(\mu) \subset$ int $D, \mathbb{P} E\left(\mu^{\prime}\right) \subset$ int $D^{\prime}$, then $D \prec D^{\prime}$.

(iv) If for $g \in \mathcal{S}$ and $\mu \in \operatorname{spec} g$ one has $\mathbb{P} E(\mu) \subset$ int $D$ for some main control set $D$, then $g \in$ int $\mathcal{S}_{\leq t}$ for some $t>0$.

4.2. Remark. Using the classification of transitive Lie groups on projective space and the theory of control sets for semigroups in semisimple Lie groups (cp. [SMT]), Barros and San Martin [BSM] were able, in certain cases, to give sharper estimates on the number of main control sets.

Theorem 4.1 suggests the following definition.

4.3. Definition. Let $D$ be a main control set of (2.8). The Floquet spectrum of the family (2.1) over $D$ is defined as

$$
\begin{aligned}
\Sigma_{F l}(D)= & \{\lambda(u, p) ;(u, p) \in \mathcal{U} \times \operatorname{int} D, u \text { is piecewise constant, periodic } \\
& \text { with period } \tau \text { such that } s(\tau, p, u)=p\},
\end{aligned}
$$

where $s(\cdot, p, u)$ denotes again the solution of $(2.8)$ with $s(0, p, u)=p$. The Floquet spectrum of the family $(2.1)$ is $\Sigma_{F l}=\bigcup_{i=1}^{k} \Sigma_{F l}\left(D_{i}\right)$.

\subsection{Remark.}

(i) Note that $(u(\cdot), s(\cdot, p, u)) \tau$-periodic with $p \in \operatorname{int} D$ implies that $s(t, p, u) \in$ int $D$ for all $t \in \mathbb{R}$.

(ii) According to Theorem 4.1 the periodic spectrum over a main control set $D$ can be written as

$\Sigma_{F l}(D)=\left\{\lambda \in \mathbb{R} ;\right.$ there exists $g \in$ int $\mathcal{S}_{\leq t}$ and $\mu \in \operatorname{spec} g$ such that

$$
\left.\mathbb{P} E(\mu) \subset \operatorname{int} D \text { and } \lambda=\frac{1}{\tau(g)} \log |\mu|\right\},
$$

where $\tau(g)=\sum_{j=1}^{n} t_{j}$ for $g=\exp \left(t_{n} B_{n}\right) \cdots \cdot \exp \left(t_{1} B_{1}\right)$. This means that $\Sigma_{F l}(D)$ is defined through those Floquet exponents of the $g \in$ int $\mathcal{S}$ whose projected (generalized) eigenspaces are contained in int $D$. In particular, for each $g \in$ int $\mathcal{S}$ there exists $p \in$ int $D$ such that $\lambda\left(u_{g}, p\right) \in \Sigma_{F l}(D)$, where $u_{g} \in \mathcal{U}$ is a periodic perturbation with fundamental matrix $g$.

(iii) The main control sets $D \subset \mathbb{P}^{d-1}$ uniquely correspond to the topologically transitive components $\mathcal{D}$ of the associated flow $\mathbb{P} \Phi$ on $\mathcal{U} \times \mathbb{P}^{d-1}$ with int $\pi_{\mathbb{P}} \mathcal{D} \neq$ $\phi$ via

$$
\mathcal{D}=c \ell\left\{(u, p) \in \mathcal{U} \times \mathbb{P}^{d-1} ; \varphi(t, p, u) \in \operatorname{int} D \text { for all } t \in \mathbb{R}\right\}
$$

(see [CKf], Theorem 3.9). Hence the Floquet spectrum is defined via topologically transitive components of the flow $\Phi$, while the Morse spectrum is defined via the chain recurrent components.

4.5. Theorem. Let $(\mathrm{H})$ be satisfied. Consider the main control sets $D_{1}, \ldots, D_{k}$ of the system (2.8) in $\mathbb{P}^{d-1}$.

(i) For each $i \in\{1, \ldots, k\}, c l \Sigma_{F l}\left(D_{i}\right)$ is a bounded interval.

(ii) If $D_{i} \prec D_{j}$, then $\Sigma_{F l}\left(D_{i}\right) \leq \Sigma_{F l}\left(D_{j}\right)$ in the sense that inf $\Sigma_{F l}\left(D_{i}\right) \leq$ $\inf \Sigma_{F l}\left(D_{j}\right)$ and $\sup \Sigma_{F l}\left(D_{i}\right) \leq \sup \Sigma_{F l}\left(D_{j}\right)$. 
(iii) $\sup \Sigma_{F l}\left(D_{k}\right)=\sup \Sigma_{F l}=\max \Sigma_{L y}$ and inf $\Sigma_{F l}\left(D_{1}\right)=\inf \Sigma_{F l}=\min \Sigma_{L y}$.

Proof.

(i) Abbreviate $D=D_{i}$ and let $j=1,2, \lambda_{j} \in \Sigma_{F l}(D)$ with corresponding points $\left(u_{j}, p_{j}\right) \in \mathcal{U} \times \operatorname{int} D$, periods $\tau_{j} \geq 0$ and fundamental matrices $g_{j}\left(\tau_{j}\right)$. Since $\left\{s\left(t, p_{j}, u_{j}\right) ; t \in \mathbb{R}\right\}$ are compact subsets of the interior of the control set $D$, there exist $T>0$ and $v_{j} \in \mathcal{U}$, piecewise constant, with $s\left(t_{1}, p_{1}, v_{1}\right)=p_{2}$ and $s\left(t_{2}, p_{2}, v_{2}\right)=p_{1}$ for some $t_{1}, t_{2} \leq T$. For $m, n \in \mathbb{N}$ define a control $u^{m, n}$ via concatenation on the time interval $\left[0, t^{m, n}\right]$ with $t^{m, n}=m \tau_{1}+t_{1}+n \tau_{2}+t_{2}$ as

$$
u^{m, n}=v_{2} \cdot \underbrace{u_{2} \cdots u_{2}}_{n \text {-times }} \cdot v_{1} \cdot \underbrace{u_{1} \cdots \cdots u_{1}}_{m \text {-times }},
$$

and on $\mathbb{R}$ as the $t^{m, n}$-periodic continuation. Assume without loss of generality that $\lambda_{1}<\lambda_{2}$, and choose $\lambda \in\left[\lambda_{1}, \lambda_{2}\right]$ and $\varepsilon>0$. Then there exist $\bar{m}, \bar{n} \in \mathbb{N}$ such that $s\left(\cdot, p_{1}, u^{\bar{m}, \bar{n}}\right)$ is $t^{\bar{m}, \bar{n}}$-periodic with $\left|\lambda-\lambda\left(u^{\bar{m}, \bar{n}}, p_{1}\right)\right|<\varepsilon$. Note that by assumption $(\mathrm{H})$ the controls $v_{i}$ can be chosen in such a way that the corresponding fundamental matrices satisfy $g_{v_{i}}\left(t_{i}\right) \in$ int $\mathcal{S}_{\leq t_{i}}$ for $i=1,2$. Hence the fundamental matrix $g_{\bar{m}, \bar{n}}$ corresponding to $u^{\bar{m}, \bar{n}}$ satisfies $g_{\bar{m}, \bar{n}}\left(t^{\bar{m}, \bar{n}}\right) \in$ int $\mathcal{S}_{\leq t^{\bar{m}, \bar{n}}}$ and thus $\Sigma_{F l}(D)$ is dense in $\left[\lambda_{1}, \lambda_{2}\right]$.

(ii) The inequalities follow directly from the construction of the main control sets in the proof of Theorem 3.10 (ii) in [CKg].

(iii) The fact that $\sup \Sigma_{L y}$ and inf $\Sigma_{L y}$ are actually Lyapunov exponents follows from Theorem 3.1. From part (ii) we know that $\sup \Sigma_{F l}=\sup \Sigma_{F l}\left(D_{k}\right)$ and $\inf \Sigma_{F l}=\inf \Sigma_{F l}\left(D_{1}\right)$. The supremum and the infimum of the Floquet spectrum can be characterized as the extremal exponential growth rates of the spectral radius and the spectral coradius: Define for $g \in G \ell(d, \mathbb{R}), \quad r(g)=$ $\max \{|\mu| ; \mu \in \operatorname{spec} g\}$ and $\operatorname{cor}(g)=\min \{|\mu|\} ; \mu \in \operatorname{spec} g\}$. By Theorem 4.1 in [CKe] we have that

$$
\sup \Sigma_{F l}=\limsup _{t \rightarrow \infty} \frac{1}{t} \sup _{g \in \mathcal{S}_{t}} \log r(g) \text { and } \inf \Sigma_{F l}=\limsup _{t \rightarrow \infty} \frac{1}{t} \inf _{g \in \mathcal{S}_{t}} \log \operatorname{cor}(g)
$$

and the right-hand sides of the above equalities are the extremal Lyapunov exponents. This yields the result.

4.6. Remark. Part (i) of Theorem 4.5 can be strengthened in the following way: for a main control set $D$ define its multiplicity as (compare Remark 3.16 in [CKg])

$$
\begin{array}{r}
m(D)=\#\{\lambda \in \mathbb{R} ; \lambda \text { is an eigenvalue of } g, \text { with } \mathbb{P} E(\lambda) \subset \text { int } D\}, \\
\text { where } g \in \text { int } \mathcal{S}_{\leq t}, t>0 .
\end{array}
$$

This is well defined since this number does not depend on $g \in \bigcup_{t>0}$ int $\mathcal{S}_{\leq t}$. Then $c \ell \Sigma_{F l}(D)$ coincides with $\Sigma_{F l}(D)$, except for maybe at most $m(D)+1$ points.

For a proof note that if $\lambda_{i} \in \Sigma_{F l}(D)$ with fundamental matrices $g_{i} \in$ int $\mathcal{S}_{\leq t_{i}}, i=$ 1,2 , then there exists a continuous path $g:\left[0, t_{1}+t_{2}\right] \rightarrow$ int $\mathcal{S}_{\leq t_{1}+t_{2}}$ with $g(0)=g_{1}$ and $g\left(t_{1}+t_{2}\right)=g_{2}$, compare Proposition 2.1 (iv) in [CKg]. Along this path the eigenvalues vary continuously and the projections of the corresponding eigenspaces are contained in the appropriate main control sets. Hence there are at most $m(D)-1$ points in $\operatorname{int}\left(c \ell \Sigma_{F l}(D)\right)$ that are not in the Floquet spectrum over $D$. Furthermore, the two endpoints of $c \ell \Sigma_{F l}(D)$ need not be contained in $\Sigma_{F l}(D)$, compare Example $4.7(\mathrm{i})$. 


\subsection{Remarks and Examples.}

(i) The Floquet spectral interval $\Sigma_{F l}(D)$ is, in general, not closed, because it is defined over the interior of $D$. Example 3.2 illustrates this point: the control sets for this example are given by $D_{1}=$ int $E_{1}$ and $D_{2}=E_{2}$. The corresponding intervals of the Floquet spectrum are $\Sigma_{F l}\left(D_{1}\right)=\left(-1, \frac{3}{2}\right)$ and $\Sigma_{F l}\left(D_{2}\right)=\left(\frac{1}{2}, 3\right)$, they consist of the (real) eigenvalues corresponding to eigendirections in int $D_{1}$ and int $D_{2}$.

(ii) The same Example 3.2 shows that different intervals of the periodic spectrum can overlap.

(iii) The spectrum of the constant matrices of the family (2.1) may be strictly contained in the Floquet spectrum; consider the linear oscillator with perturbation in the restoring force

$$
\ddot{y}+2 b \dot{y}+(1+u(t)) y=0
$$

where $u(t) \in[-1,1]$. Setting $\left(x_{1}, x_{2}\right)=(y, \dot{y})$ we can write

$$
\dot{x}=\left\{\left(\begin{array}{rc}
0 & 1 \\
-1 & -2 b
\end{array}\right)+u(t)\left(\begin{array}{rr}
0 & 0 \\
-1 & 0
\end{array}\right)\right\} x \quad \text { in } \mathbb{R}^{2} .
$$

For $b^{2}<\sqrt{2}$ the projected system on $\mathbb{P}^{1}$ has a unique main control set $D=\mathbb{P}^{1}$, compare e.g. [CKe], Section 6. For $b=\frac{1}{4}$, the spectrum of the constant matrices of $(4.3)$ is the interval $\left[-\frac{1}{2}, 0\right]$. On the other hand, by Theorem 4.5, the Floquet spectrum consists of one interval $\Sigma_{F l}$, and by Theorem 4.5 (iii) we have that $c \ell \Sigma_{F l}=c \ell \Sigma_{L y}$. The results in Section 6 of [CKe] show that $\inf \Sigma_{L y}<-\frac{1}{2}$ and $\sup \Sigma_{L y}>0$, hence $\left[-\frac{1}{2}, 0\right] \varsubsetneqq \Sigma_{F l}$. In fact, there exists a critical value $b_{0} \sim 0.405$ such that for all $b$ with $b^{2}<b_{0}^{2}$ the constant spectrum is strictly contained in the Floquet spectrum, while for all $b$ with $b^{2}>b_{0}^{2}$ the two spectra coincide.

4.8. Corollary. Assume that we are given a family of matrices (2.1) such that $(\mathrm{H})$ holds and that the system (2.8) has exactly one main control set $D \subset \mathbb{P}^{d-1}$. Then

$$
c \ell \Sigma_{F l}=\Sigma_{L y}=\Sigma_{M o}
$$

up to at most $d-1$ points in the interior of this interval.

Proof. If $D \subset \mathbb{P}^{d-1}$ is the only main control set of the system (2.8), then $D=\mathbb{P}^{d-1}$ and hence $\mathbb{P}^{d-1}$ is the unique chain control set. Therefore $\mathcal{U} \times \mathbb{P}^{d-1}$ is the chain recurrent set of the projected flow (2.10). Thus the Floquet spectrum and the Morse spectrum each consist of one interval, and we have $\Sigma_{F l} \subset \Sigma_{L y} \subset \Sigma_{M o}$. By Theorem 3.1(ii) we know that $\max \Sigma_{M o}=\max \Sigma_{L y}$, and by Theorem 4.5(iii) it holds that $\max \Sigma_{L y}=\sup \Sigma_{F l}$. Analogous equalities are true for the minimal exponents. This, together with Remark 4.6 proves the result.

Corollary 4.8 shows that for systems with unique main control set the Lyapunov spectrum is nothing but the Floquet spectrum together with the endpoints of the Floquet spectral interval, and at the same time this set coincides with the interval of the Morse spectrum. As the following example shows, the intervals of the periodic spectrum and of the Morse spectrum need not be the same, if more than one main control set exists. 
4.9. Example. Consider the family of matrices

$$
\left(\begin{array}{cc}
1 & \frac{1}{4} \\
\frac{1}{4} & \frac{3}{2}
\end{array}\right)+u_{1}(t)\left(\begin{array}{ll}
0 & 1 \\
1 & 0
\end{array}\right)+u_{2}(t)\left(\begin{array}{ll}
0 & 0 \\
0 & 1
\end{array}\right)
$$

with $U=\left[-\frac{1}{4}, \frac{1}{4}\right] \times\left[-\frac{1}{2}, \frac{1}{2}\right]$. Parametrize the projective space $\mathbb{P}^{1}$ via the angle $\theta$ as $\mathbb{P}^{1}=\left\{\theta ;-\frac{\pi}{2}<\theta \leq \frac{\pi}{2}\right\}$, then the two main control sets are given in terms of $\theta$ as $D_{1}=\left(-\frac{\pi}{4}, 0\right), D_{2}=\left[\frac{\pi}{4}, \frac{\pi}{2}\right]$. The unique chain control set is $E=\mathbb{P}^{1}$, compare Example 5.8 in $[\mathrm{CKg}]$. We compute the intervals of the Floquet spectrum as

$$
\Sigma_{F l}\left(D_{1}\right)=\left(\frac{1}{2}, 1\right), \quad \Sigma_{F l}\left(D_{2}\right)=\left(1, \frac{3}{2}+\frac{1}{2} \sqrt{2}\right)
$$

from the eigenvalues of the constant matrices in (4.4) as before.

Hence by Theorem 3.1 there is exactly one interval of the Morse spectrum and $\Sigma_{M o}=\Sigma_{L y}=\left[\frac{1}{2}, \frac{3}{2}+\frac{1}{2} \sqrt{2}\right]$.

For families of $2 \times 2$ matrices the situations in Remark 4.7, Corollary 4.8 and Example 4.9 are the only ones possible.

4.10. Corollary. Let $d=2$ and assume $(\mathrm{H})$. Then $\mathrm{cl} \Sigma_{F l}=\Sigma_{L y}=\Sigma_{M o}$. More precisely, we have the following three possibilities.

(i) The projected system (2.8) has two main control sets $D_{1} \prec D_{2}$ and two chain control sets $E_{1}=c l D_{1}, E_{2}=D_{2}$, then

$$
c \ell \Sigma_{F l}=\bigcup_{i=1}^{2} c \ell \Sigma_{F l}\left(D_{i}\right)=\Sigma_{L y}=\bigcup_{j=1}^{2} \Sigma_{M o}\left(E_{j}\right),
$$

and the entire spectrum consists of real eigenvalues of constant matrices.

(ii) The projected system has two main control sets $D_{1} \prec D_{2}$, but one chain control set $E=\mathbb{P}^{1}$, then

$$
c \ell \Sigma_{F l}=\bigcup_{i=1}^{2} c \ell \Sigma_{F l}\left(D_{i}\right)=\Sigma_{L y}=\Sigma_{M o}
$$

again, the entire spectrum consists of real eigenvalues of constant matrices.

(iii) The projected system has one main control set $D=\mathbb{P}^{1}$, hence the chain control set is $E=\mathbb{P}^{1}$, then

$$
c \ell \Sigma_{F l}(D)=\Sigma_{L y}=\Sigma_{M o}
$$

and the spectrum of constant matrices may be contained strictly in $\Sigma_{L y}$.

Proof. It follows from [CKg], Theorem 5.6 that the cases (i)-(iii) are the only ones possible.

(i) In this case we know from [Jo], Theorem 4.1 that $c \ell \Sigma_{F l}=\bigcup_{i=1}^{2} c \ell \Sigma_{F l}\left(D_{i}\right)=$ $\Sigma_{L y}$ and $\Sigma_{L y}$ consists of real eigenvalues of constant matrices. By Theorem 3.1 we have that $\Sigma_{L y} \subset \Sigma_{M o}$ and that $\sup \Sigma_{M o}$ and inf $\Sigma_{M o}$ are actually Lyapunov exponents. Hence the result.

(ii) If there are two main control sets $D_{1} \prec D_{2}$, but only one chain control set $E$ (which then necessarily equals $\mathbb{P}^{1}$ ), then either $c \ell D_{1} \cap D_{2} \neq \phi$ or there exists a constant matrix $A(u), u \in U$ in the family (2.1) with a double real eigenvalue $\lambda$ such that $\mathbb{P} E(\lambda)=\mathbb{P}^{1}$. In both cases we have $c \ell \Sigma_{F l}\left(D_{1}\right) \cap c \ell \Sigma_{F l}\left(D_{2}\right) \neq$ 
$\phi$. Again we have $\Sigma_{L y}=\bigcup_{i=1}^{2} c \ell \Sigma_{F l}\left(D_{i}\right)$, which in this case is one interval consisting of real eigenvalues of constant matrices. Hence $\Sigma_{L y}=\Sigma_{M o}$ again by Theorem 3.1.

(iii) This follows directly from Theorems 3.1 and 4.5 , since $\Sigma_{F l}$ and $\Sigma_{M o}$ are intervals with the same boundary points. Example 4.7 (iii) shows that the constant spectrum may be strictly contained in the Lyapunov spectrum.

The situation in the 2-dimensional case is simple, because the control sets of (2.8) on the one-dimensional space $\mathbb{P}^{1}$ are given by the eigendirections of the constant matrices in a given family, compare also [CKc] for a general discussion of control sets in one dimensional spaces. For $d \geq 3$ this need not be true, and we have to analyze which Lyapunov exponents $\lambda(u, x)$ can be represented in the periodic spectrum. For this we have to consider the limit sets of trajectories of (2.8): for $(u, p) \in \mathcal{U} \times \mathbb{P}^{d-1}$ define the $\omega$-limit set by

$$
\begin{aligned}
\omega(u, p)= & \left\{(v, q) \in \mathcal{U} \times \mathbb{P}^{d-1} ; \text { there exists a sequence } t_{k} \rightarrow \infty\right. \text { such } \\
& \text { that } \left.\lim _{k \rightarrow \infty} \mathbb{P} \Phi\left(t_{k}, u, p\right)=(v, q)\right\} \subset \mathcal{U} \times \mathbb{P}^{d-1},
\end{aligned}
$$

and $\pi_{\mathbb{P}} \omega(u, p) \subset \mathbb{P}^{d-1}$ is its projection onto $\mathbb{P}^{d-1}$.

The following result is the starting point of the discussion in the next section, where we analyze the connections between the Floquet and the Morse spectrum via 'closing of chains'.

4.11. Theorem. Assume $(\mathrm{H})$ and let $D \subset \mathbb{P}^{d-1}$ be a main control set of (2.8). Consider a point $(u, p) \in \mathcal{U} \times \mathbb{P}^{d-1}$ such that $\pi_{\mathbb{P}} \omega(u, p) \subset D$, and there is $t_{0} \geq 0$ with $s\left(t_{0}, p, u\right) \in$ int $D$.

Then $\lambda(u, p) \in c \ell \Sigma_{F l}(D)$.

Proof. We proceed in two steps. First we show that $\lambda(u, p)$ for points $(u, p)$ as in the formulation of the theorem can be approximated by Lyapunov exponents of periodic $u \in \mathcal{U}$ with periodic trajectory in int $D$. Then we show that the exponents of arbitrary periodic $u \in \mathcal{U}$ with periodic trajectory in int $D$ are in the closure of the Floquet spectrum over $D$.

(a) Let $(u, p) \in \mathcal{U} \times \mathbb{P}^{d-1}$ be a point with $\pi_{\mathbb{P}} \omega(u, p) \subset D$ and $s\left(t_{0}, p, u\right) \in$ int $D$. Hence, without loss of generality, we may assume that $p \in$ int $D$. Let $K \subset D$ be a compact set such that

$$
\pi_{\mathbb{P}} \omega(u, p) \cap \text { int } D \subset \text { int } K \text { and } \pi_{\mathbb{P}} \omega(u, p) \cap(D \backslash \text { int } D) \subset \text { int }_{D \backslash \text { int }}{ }_{D} K \text {. }
$$

Such a set $K$ exists because $D \backslash$ int $D$ is open in $\partial D$, and $\pi_{\mathbb{P}} \omega(u, p)$ is compact. For $x \in \mathbb{P}^{d-1}$ and $t>0$ let $\mathcal{O}_{\leq t}^{+}(x):=\left\{y \in \mathbb{P}^{d-1}\right.$; there are $0 \leq \tau \leq t$ and $u \in \mathcal{U}$ with $y=s(\tau, x, u)\}$.

Define $\tau:=\inf \left\{t \geq 0 ; p \in \mathcal{O}_{\leq t}^{+}(x)\right.$ for all $\left.x \in K\right\}$. By Proposition 2.3 in [CKa] we know that $\tau<\infty$.

Fix $\varepsilon>0$ and let $\left\{t_{n}, n \in \mathbb{N}\right\}$ be a sequence with $t_{n} \rightarrow \infty$ and $\lambda(u, p)=$ $\lim _{n \rightarrow \infty} \frac{1}{t_{n}} \int_{0}^{t_{n}} q(u(\sigma) s(\sigma)) d \sigma$, compare (2.8). Pick $n_{1} \in \mathbb{N}$ such that 


$$
\frac{\tau}{t_{n_{1}}+\tau} \max _{U \times \mathbb{P}^{d-1}}|q(u, p)|<\frac{\varepsilon}{2}
$$

and $n_{2} \in \mathbb{N}$ such that

$$
\left|\frac{1}{t_{n_{2}}+\tau} \int_{0}^{t_{n_{2}}} q(u(\sigma), s(\sigma)) d \sigma-\lambda(u, p)\right|<\frac{\varepsilon}{2} \text { for all } n \geq n_{2} .
$$

Let $N=\max \left\{n_{1}, n_{2}\right\}$, and choose a control $v:[0, T] \rightarrow U$ with $T \leq \tau$ and $s\left(T, s\left(t_{N}, p, u\right), v\right)=p$. Define a new control $w$ via concatenation on $\left[0, t_{N}+T\right]$ as $w=v \circ u$, and continue $t_{N}+T$-periodically. We have

$$
|\lambda(u, p)-\lambda(w, p)|<\varepsilon
$$

and since $s\left(t_{N}, p, u\right) \in$ int $D$, the first claim follows.

(b) Let $(u, p) \in \mathcal{U} \times \mathbb{P}^{d-1}$ be a periodic point of the flow $\Phi$ with period $T \geq 0$, and assume that $s(t, p, u) \in$ int $D$ for all $t \in \mathbb{R}$. Let $K \subset$ int $D$ be a compact set with $s(t, p, u) \in$ int $K$ for all $t \in \mathbb{R}$. Define again the first hitting time $\tau$ as above, and choose $\varepsilon>0$. Since $(u, p)$ is a $T$-periodic point of $\Phi$, we have $\lambda(u, p)=\frac{1}{T} \int_{0}^{T} q(u, s) d \tau$. Choose $N \in \mathbb{N}$ such that $\frac{\tau}{N T+\tau} \max _{U \times \mathbb{P}^{d-1}}|q(u, p)|<\frac{\varepsilon}{2}$. On the compact time interval $[0, N T]$ we find a piecewise constant control $v$ such that

$$
\left|\int_{0}^{N T} q(u(\tau), s(\tau, p, u)) d \tau-\int_{0}^{N T} q(v(\tau), s(\tau, p, v)) d \tau\right|<\frac{1}{2} \varepsilon N T
$$

and $s(N T, p, v) \in K$. Since $K \subset$ int $D$ there exists a control $w:[0, \sigma] \rightarrow$ $U$, piecewise constant, with $\sigma \leq \tau$ and $s(\sigma, s(N T, p, v), w)=p$. Now the concatenation $\left.w \circ u\right|_{[0, N T]}$ solves the problem.

According to Theorem 4.11 the difference between the Morse and the Floquet spectrum can be made up of those chains whose limit is contained in chain control sets, but not in the interior of control sets. To close this gap, we have to be able to 'close chains', i.e. to find trajectories of the system (2.8) in the interior of some control set with Lyapunov exponents close to the chain exponent. This will be accomplished in the next section by embedding the system (2.8) into a family of systems with varying perturbation range.

4.12. Remark. It has been shown above in Corollary 4.10 (ii) and Example 4.9 that the decomposition of the Lyapunov spectrum $\Sigma_{L y}$ into the closures of the intervals of the Floquet spectrum may be finer than the Morse spectrum. However, there need not be a (constant dimensional) subbundle decomposition of $\mathcal{U} \times \mathbb{R}^{d}$ corresponding to the intervals of the Floquet spectrum that respects the (generalized) eigenspaces of even the constant matrices in the family (2.1). Example 4.9 illustrates this point: For $u_{1}=-\frac{1}{4}, u_{2}=-\frac{1}{2}$ the resulting matrix $\left(\begin{array}{ll}1 & 0 \\ 0 & 1\end{array}\right)$ has a double real eigenvalue $\lambda=1$ with eigenspace $E(\lambda)=\mathbb{R}^{2}$. Its projection $\mathbb{P} E(\lambda)$ intersects int $D_{1}$ and int $D_{2}$. On the other hand, chain control sets always lead to subbundle decompositions of $\mathcal{U} \times \mathbb{R}^{d}$ via the chain recurrent components $\mathbb{P} \mathcal{E}_{j}$ of $\mathbb{P} \Phi$, compare 
Theorem 3.1(iii). Therefore, it is natural to base the decomposition of $\Sigma_{L y}$ on the intervals of the Morse spectrum, which will be our point of view in the next section.

\section{The Lyapunov Spectrum}

In order to express the exponents in the Morse spectrum as actual Lyapunov exponents, we need to be able to approximate the corresponding chains by trajectories of the projected system (2.8) in such a way that the Lyapunov exponents approximate the chain exponents. Theorem 4.11 shows that approximation of the growth rates is possible for trajectories whose $\omega$-limits set intersects the interior of some control set. Furthermore, $\pi_{\mathbb{P}} \omega(u, p) \subset$ int $D$ for some control set $D$ if and only if $\omega(u, p)$ consists of 'inner pairs', compare Theorem 4.4 in [CKh]. Hence the inner pair condition guarantees that chains can be closed. (For a set up, in which the closing of chains follows from Bowen's Shadowing Lemma, see [CKj], Section 6.)

The natural way to formulate an inner pair condition in the present context is by embedding the set of matrices (2.1) into a family of systems with varying control range. This guarantees that we can obtain the desired characterization of the Lyapunov spectrum for almost all perturbation ranges.

For $U \subset \mathbb{R}^{m}$ compact and convex with $0 \in$ int $U$ denote

$$
U^{\rho}:=\rho \cdot U=\{\rho \cdot u ; u \in U\} \text { for } \rho \geq 0 .
$$

To complete the picture we also consider the case of unbounded perturbations. Let $\mathcal{U}^{\infty}=\bigcup_{\rho>0} \mathcal{U}^{\rho}=L^{\infty}\left(\mathbb{R}, \mathbb{R}^{m}\right)$ and denote the corresponding perturbed system by $\left(2.1^{\infty}\right)$.

All quantities defined in Sections 2-4 will be written with a superscript $\rho$ to indicate their dependence on the control range $U^{\rho}$ for $0 \leq \rho \leq \infty$. Note that for $\rho=0$ we obtain the unperturbed matrix $A_{0}$.

We continue to assume the nondegeneracy condition $(\mathrm{H})$ in the form

$$
\operatorname{dim} \mathcal{L} \mathcal{A}\left\{h(\cdot, u) ; u \in U^{\rho}\right\}(p)=d-1 \text { for all } p \in \mathbb{P}^{d-1}, \text { all } \rho>0 .
$$

Since $0 \in$ int $U$, we have that if $\left(\mathrm{H}^{\rho}\right)$ holds for some $\rho>0$, then it holds for all $\rho>0$. According to the results of Section 3 we need the inner pair condition for all $(u, p) \in \mathcal{U} \times \mathbb{P}$ with $\omega(u, p) \subset \mathcal{E}_{j}$ for some $j=1 \ldots \ell$. But every $\omega$-limit set is contained in one of the chain recurrent components of the flow $\mathbb{P} \Phi$. Hence we will assume from now on for the family of systems $\left(2.1^{\rho}\right), \rho \geq 0$ the following $\rho-\rho^{\prime}$ inner pair condition.

For all $0 \leq \rho<\rho^{\prime}$ and all $(u, p) \in \mathcal{U}^{\rho} \times \mathbb{P}^{d-1}$ there exist $T>0$

$$
\text { and } S>0 \text { such that } s(T, p, u) \in \text { int } \mathcal{O}_{\leq T+S}^{\rho^{\prime}+}(p) \text {, }
$$

where $s(\cdot, p, u)$ denotes again the solution of $(2.8)$ and $\mathcal{O}_{\leq T+S}^{\rho^{\prime}+}(p)=\{q \in \mathbb{P}$; there is $v \in \mathcal{U}^{\rho^{\prime}}$ with $s(t, p, v)=q$ for some $\left.0 \leq t \leq T+S\right\}$.

We first study the behavior of main and chain control sets of the family of systems $\left(2.8^{\rho}\right)$ under the $\rho-\rho^{\prime}$ inner pair condition (I). Let $\lambda_{1}, \ldots, \lambda_{k}$ be the different real parts of eigenvalues of the unperturbed matrix $A_{0}$, and let $E\left(\lambda_{i}\right), i=1, \ldots, k \leq d$ the corresponding (sums of generalized) eigenspaces. The sets $\mathbb{P} E\left(\lambda_{i}\right) \subset \mathbb{P}^{d-1}$ are the Morse sets (i.e. the components of the chain recurrent set) of the projected flow of $\dot{x}=A_{0} x$ on $\mathbb{P}^{d-1}$. 
Let $\mathcal{C}\left(\mathbb{P}^{d-1}\right)$ denote the set of compact subsets of $\mathbb{P}^{d-1}$ with the Hausdorff metric, and consider for $i=1, \ldots, k$ the following maps:

$$
\begin{gathered}
D_{i}:[0, \infty] \rightarrow \mathcal{C}\left(\mathbb{P}^{d-1}\right), \quad \rho \mapsto c \ell D_{i}^{\rho} \\
\text { with } D_{i}^{0}=\mathbb{P} E\left(\lambda_{i}\right) \text { and } \mathbb{P} E\left(\lambda_{i}\right) \subset \text { int } D_{i}^{\rho},
\end{gathered}
$$

$$
\begin{array}{r}
E_{i}:[0, \infty] \rightarrow \mathcal{C}\left(\mathbb{P}^{d-1}\right), \quad \rho \mapsto E_{i}^{\rho} \\
\text { with } E_{i}^{0}=\mathbb{P} E\left(\lambda_{i}\right) \text { and } \mathbb{P} E\left(\lambda_{i}\right) \subset E_{i}^{\rho} .
\end{array}
$$

Furthermore, let

$$
\begin{aligned}
& k(\cdot):[0, \infty] \rightarrow\{1, \ldots k\} \text { denote for } \rho>0 \text { the number of (different) } \\
& \text { main control sets and set } k(0):=k .
\end{aligned}
$$

The following lemma shows in particular, that under assumption (I) the maps (5.2) and (5.3) are well defined.

\subsection{Lemma.}

(i) For all $i=1, \ldots, k$ and all $0 \leq \rho \leq \infty$ there are unique main control sets $D_{i}(\rho)$ and chain control sets $E_{i}(\rho)$ satisfying the conditions in (5.2) and (5.3), respectively.

(ii) For all $i=1, \ldots, k$ and all $0 \leq \rho<\rho^{\prime} \leq \infty$

$$
D_{i}(\rho) \subset E_{i}(\rho) \subset \text { int } D_{i}\left(\rho^{\prime}\right) .
$$

Proof.

(i) The assertion for the chain control sets is obvious (naturally, some of the $E_{i}(\rho)$ may coincide). Using (I) with $\rho=0$, one finds by Theorem 4.4 in [CKh] unique main control sets $D_{i}^{\rho^{\prime}}, \rho^{\prime}>0$, satisfying the conditions in (5.2).

(ii) By Theorem 5.5 in $[\mathrm{CKg}]$ we know that each chain control set contains a main control set, i.e. the first inclusion follows. Applying (I) and again Theorem 4.4 in $[\mathrm{CKh}]$ implies the second inclusion.

The following theorem characterizes the relations between the main control sets and the chain control sets.

5.2. Theorem. Assume $\left(\mathrm{H}^{\rho}\right)$ and the $\rho-\rho^{\prime}$ inner pair condition (I). For $i=$ $1, \ldots, k$, the maps $D_{i}(\rho)$ and $E_{i}(\rho), 0 \leq \rho \leq \infty$, defined in (5.2) and (5.3), respectively, have the following properties.

(i) $D_{i}(\rho)$ and $E_{i}(\rho)$ are strictly increasing in $\rho$.

(ii) $D_{i}(\rho)$ is left continuous and $E_{i}(\rho)$ is right continuous in $\rho$.

(iii) There exist at most finitely many values $\rho_{i}^{1}<\cdots<\rho_{i}^{n(i)}$ such that the maps $D_{i}(\rho)$ and $E_{i}(\rho)$ are discontinuous at $\rho=\rho_{i}^{n}, n=1, \ldots, n(i)$.

(iv) For each $\rho \neq \rho_{i}^{n}, n=1, \ldots, n(i)$, we have $c \ell D_{i}(\rho)=E_{i}(\rho)$, while for $\rho=\rho_{i}^{n}$ there are at least two main control sets $D_{i_{1}}^{\rho}$ and $D_{i_{2}}^{\rho}$ with $D_{i_{1}}^{\rho} \cup D_{i_{2}}^{\rho} \subset E_{i}^{\rho}$.

(v) The map $k(\cdot)$ defined in (5.4) is decreasing with jumps exactly at those $\rho$ for which there exist $i$ and $n$ with $\rho=\rho_{i}^{n}$. In particular, $n(i) \leq k-k(\infty)$ and there exist $\rho_{0}>0$ and $\rho_{\infty}<\infty$ such that $k(\rho)=k$ for $\rho \in\left[0, \rho_{0}\right]$ and $k(\rho)=k(\infty)$ for $\rho \in\left[\rho_{\infty}, \infty\right]$. 
Proof.

(i) This follows from (5.5), since chain control sets are closed.

(ii) Right continuity of chain control sets was proved in Theorem 5.1 of [CKh]. Left continuity of the main control sets follows from standard compactness arguments and continuous dependence of the solutions of differential equations on the right hand side:

Fix $i \in\{1, \ldots k\}$ and abbreviate $D(\rho):=D_{i}(\rho)$. We will show: if $K \subset$ int $D(\bar{\rho})$ is a compact set with $d(K, \operatorname{cl} D(\bar{\rho}))<2 \varepsilon$, then there exists $\delta>0$ such that $K \subset$ int $D(\rho)$ for all $\bar{\rho}-\delta<\rho<\bar{\rho}$. This will imply left continuity, since $c \ell D(\bar{\rho})=$ cl int $D(\bar{\rho})$ by $(\mathrm{H})$.

Fix $x \in K$ and consider a piecewise constant $u \in \mathcal{U}^{\bar{\rho}}$ and $T>0$ with $u(t)=$ $u^{i} \in U^{\bar{\rho}}$ for $t \in\left[t_{0}+\cdots+t_{i-1}, t_{0}+\cdots+t_{i}\right), i=1 \ldots n$, where $\sum_{i=1}^{n} t_{i}=T$ and $t_{0}=0, t_{i}>0$. By $\left(H^{\rho}\right)$ we may choose $T>0$ arbitrarily small, and $u \in \mathcal{U}^{\bar{\rho}}$ such that the map $\psi: \mathbb{R}^{n} \rightarrow \mathbb{P}^{d-1},\left(\tau_{1}, \ldots, \tau_{n}\right) \mapsto s\left(\sum_{i=1}^{n} \tau_{i}, x, u^{\tau}\right)$ has rank $d-1$ at $\tau=\left(\tau_{1}, \ldots, \tau_{n}\right)=\left(t_{1}, \ldots, t_{n}\right)$; here $u^{\tau}$ is the control that coincides on $\left[\tau_{1}+\right.$ $\left.\cdots+\tau_{i-1}, \tau_{1}+\cdots+\tau_{i}\right)$ with $u^{i}$. Choose $T>0$ small enough such that $y=$ $s(T, x, u) \in$ int $D^{\bar{\rho}}$. The Implicit Function Theorem, applied to the map $(0, \infty) \times$ $\mathbb{R}^{n} \rightarrow \mathbb{P}^{d-1},\left(\rho, \tau_{1}, \ldots, \tau_{n}\right) \mapsto s\left(\sum_{i=1}^{n} \tau_{i}, x, \frac{\rho}{\bar{\rho}} u^{\tau}\right)$ yields the existence of $\delta_{0}=\delta_{0}(x)>$ 0 and of a neighborhood $N_{0}(y)$ with $N_{0}(y) \subset \mathcal{O}^{\rho+}(x)$ for all $\rho \geq \bar{\rho}-\delta_{0}$. Since we have $x \in$ int $D(\bar{\rho})$, there are $u_{2} \in \mathcal{U}^{\bar{\rho}}$ and $S>0$ such that $s\left(S, y, u_{2}\right)=x$. By continuous dependence of the solutions of differential equations on parameters, one finds a $\delta$ with $0<\delta<\delta_{0}$ and a neighborhood $N(y) \subset N_{0}(y)$ such that for all $\rho>\bar{\rho}-\delta$ there exists $y^{\rho} \in N(y)$ with $s\left(S, y^{\rho}, \frac{\rho}{\bar{\rho}} u_{2}\right)=x$. Hence $y$ is contained in a control set $D(\rho)$ for all $\rho>\bar{\rho}-\delta$. Again by $\left(H^{\rho}\right)$, int $\mathcal{O}^{\rho-}(x) \cap N(y) \neq \phi$, where $\mathcal{O}^{\rho-}(x)=\left\{z \in \mathbb{P}^{d-1}\right.$; there are $t>0$ and $u \in \mathcal{U}^{\rho}$ with $\left.x=s(t, y, u)\right\}$. Hence $x \in$ int $D(\rho)$. Now compactness of $K$ implies that $\delta$ can be chosen independently of $x \in K$.

(iii) From part (ii) and (5.4) we obtain that $D_{i}(\rho)$ and $E_{i}(\rho)$ are continuous at $\rho$ iff $c \ell D_{i}(\rho)=E_{i}(\rho)$. We show that inequality holds for at most $k-1$ points. For a main control set $D_{i}$ and a control $u_{g} \in \mathcal{U}$ corresponding to $g \in \mathcal{S}$ set

$$
D_{i}\left(u_{g}\right)=\left\{p \in \mathbb{P}^{d-1} ; s\left(t, p, u_{g}\right) \in c \ell D_{i} \text { for all } t \in \mathbb{R}\right\}
$$

and recall that the chain control sets $E_{j} \subset \mathbb{P}^{d-1}$ can be lifted to $\mathcal{U} \times \mathbb{R}^{d}$ (cf. Theorem 3.1) via

$$
\mathcal{V}_{j}=\left\{(u, x) \in \mathcal{U} \times \mathbb{R}^{d} ; s(t, \mathbb{P} x, u) \in E_{j} \text { for all } t \in \mathbb{R}\right\} .
$$

Then Theorem 5.6 in [CKg] implies that

$$
\mathcal{V}_{j}=c \ell\left\{\left(u_{g}, x\right) \in \mathcal{U} \times \mathbb{R}^{d} ; g \in \text { int } \mathcal{S}_{\leq t} \text { for some } t>0 \text { and } \mathbb{P} x \in \bigoplus_{i} D_{i}\left(u_{g}\right)\right.
$$

where the sum is taken over all $i$ with $\left.D_{i} \subset E_{j}\right\}$.

Therefore, $c \ell D_{i}(\rho)=E_{i}(\rho)$ iff there exists a unique main control set $D(\rho) \subset E_{i}(\rho)$.

In order to estimate the number of jumps, define the multiplicity of a chain control set $E_{i}$ corresponding (via Theorem 3.1) to a subbundle $\mathcal{V}_{i} \subset \mathcal{U} \times \mathbb{R}^{d-1}$ as 


$$
m\left(E_{i}\right)=\operatorname{dim} \mathcal{V}_{i}(u) \text { for some } u \in \mathcal{U} .
$$

This number does not depend on $u \in \mathcal{U}$. Recall the definition of the multiplicity $m(D)$ of main control sets $D$ in Remark 4.6 and note that for $g \in$ int $\mathcal{S}_{\leq t}$ the eigenspaces in different main control sets are linearly independent. Therefore we have: if $c \ell D_{i}(\rho) \neq E_{i}(\rho)$, then $m\left(\left(D_{i}(\rho)\right)+1 \leq m\left(E_{i}(\rho)\right)\right.$, and hence $m\left(\left(D_{i}(\rho)\right)<\right.$ $m\left(D_{i}\left(\rho^{\prime}\right)\right)$ for $\rho<\rho^{\prime}$.

Furthermore, $\sum_{i=1}^{k} m\left(D_{i}(\rho)\right)=d$ for all $\rho>0$. Thus the functions $D_{i}(\rho)$ and $E_{i}(\rho), i=1, \ldots, k$, have at most $k-k(\infty)$ discontinuities.

(iv),(v) It only remains to prove the existence of $\rho_{\infty}<\infty$ with the indicated property, i.e. $k(\rho)$ cannot jump at $\rho=\infty$. This is seen by a contradiction argument using finite time controllability in int $D_{i}(\infty), i=1, \ldots, k(\infty)$, with $L^{\infty}$ controls.

Theorem 5.2 characterizes the dependence of the main and the chain control sets of the family of systems $\left(2.8^{\rho}\right)$ on the size of the perturbation range. Examples 4.7 (iii) and 4.9 show that jumps in the functions $D_{i}(\rho), E_{j}(\rho)$, and $k(\rho)$ can actually occur. On the other hand, $k(\infty)$ need not be 1 and the number of main and chain control sets can be different for the unbounded perturbation range $U^{\infty}=\mathbb{R}^{m}$.

5.3. Example. Consider the matrices

$$
\left(\begin{array}{ll}
0 & 1 \\
2 & 0
\end{array}\right)+u_{1}(t)\left(\begin{array}{ll}
0 & 0 \\
0 & 1
\end{array}\right)
$$

with $U^{\rho}=[-\rho, \rho], \rho \geq 0$. The projected system has two main control sets for all $\rho>0$, namely

$$
\begin{aligned}
& D_{1}^{\rho}=\pi_{\mathbb{P}}\left\{\left(\begin{array}{l}
x_{1} \\
x_{2}
\end{array}\right) \in \mathbb{R}^{2} ; x_{2}=\alpha x_{1}, \alpha \in\left(-\frac{\rho}{2}-\sqrt{2+\frac{\rho^{2}}{4}}, \frac{\rho}{2}-\sqrt{2+\frac{\rho^{2}}{4}}\right)\right\}, \\
& D_{2}^{\rho}=\pi_{\mathbb{P}}\left\{\left(\begin{array}{l}
x_{1} \\
x_{2}
\end{array}\right) \in \mathbb{R}^{2} ; x_{2}=\beta x_{1}, \beta \in\left[-\frac{\rho}{2}+\sqrt{2+\frac{\rho^{2}}{4}}, \frac{\rho}{2}+\sqrt{2+\frac{\rho^{2}}{4}}\right]\right\} .
\end{aligned}
$$

For $U^{\infty}=\mathbb{R}$ there are two main control sets, but one chain control set $\mathbb{P}^{1}$. The corresponding spectral intervals are for $\rho \in[0, \infty)$

$$
\begin{aligned}
& \Sigma_{F l}\left(D_{1}^{\rho}\right)=\left(-\frac{\rho}{2}-\sqrt{2+\frac{\rho^{2}}{4}}, \frac{\rho}{2}-\sqrt{2+\frac{\rho^{2}}{4}}\right), \\
& \Sigma_{F l}\left(D_{2}^{\rho}\right)=\left(-\frac{\rho}{2}+\sqrt{2+\frac{\rho^{2}}{4}}, \frac{\rho}{2}+\sqrt{2+\frac{\rho^{2}}{4}}\right), \\
& \Sigma_{M o}\left(E_{1}^{\rho}\right)=c \ell \Sigma_{F l}\left(D_{1}^{\rho}\right), \Sigma_{M o}\left(E_{2}^{\rho}\right)=c \ell \Sigma_{F l}\left(D_{2}^{\rho}\right) .
\end{aligned}
$$

For $\rho=\infty$ we have one interval of the Morse spectrum $\Sigma_{M o}=\mathbb{R}=\bigcup_{i=1}^{2} c \ell \Sigma_{F l}\left(D_{i}^{\infty}\right)$. For the characterization of systems with unbounded perturbation range, but bounded Lyapunov spectrum see [CKe].

From the characterization of main and chain control sets in Theorem 5.2 we obtain the following result for the corresponding spectral intervals. 
5.4. Theorem. Assume $\left(\mathrm{H}^{\rho}\right)$ and $(\mathrm{I})$.

(i) For each $i=1, \ldots, k$ the maps $\rho \mapsto c \ell \Sigma_{F l}\left(D_{i}^{\rho}\right)$ are lower semicontinuous and increasing, hence left continuous.

(ii) For each $i=1, \ldots, k$, the maps $\rho \mapsto \Sigma_{M o}\left(E_{i}^{\rho}\right)$ are upper semicontinuous and increasing, hence right continuous.

(iii) The maps

$$
\begin{aligned}
& \rho \mapsto \inf \Sigma_{F l}\left(D_{1}^{\rho}\right)=\min \Sigma_{M o}\left(E_{1}^{\rho}\right) \text { and } \\
& \rho \mapsto \sup \Sigma_{F l}\left(D_{k}^{\rho}\right)=\max \Sigma_{M o}\left(E_{k}^{\rho}\right)
\end{aligned}
$$

are continuous.

(iv) For $\rho^{\prime}>\rho \geq 0$ and $i=1, \ldots, k$ we have $c \ell \Sigma_{F l}\left(D_{i}^{\rho}\right) \subset \Sigma_{M o}\left(E_{i}^{\rho}\right) \subset c \ell \Sigma_{F l}\left(D_{i}^{\rho^{\prime}}\right)$.

Proof.

(i) Monotonicity is obvious from the definitions. Lower semicontinuity can be seen as follows. Denote $\kappa_{i}(\rho)=\sup \Sigma_{F l}\left(D_{i}^{\rho}\right), \rho \geq 0$. Fix $\varepsilon>0$. Then there exist for each $\rho^{\prime}>0$ a time $t^{\prime}>0$ and a fundamental matrix $g^{\prime} \in$ int $\mathcal{S}_{\leq t^{\prime}}^{\rho^{\prime}}$ (denoting the systems semigroup for $\left(2.1^{\rho^{\prime}}\right)$ ) such that there is $\lambda^{\prime} \in \operatorname{spec} g^{\prime}$ with

$$
\kappa_{i}\left(\rho^{\prime}\right) \leq \frac{1}{t^{\prime}} \log \left|\lambda^{\prime}\right|+\varepsilon \quad \text { and } \quad \mathbb{P} E\left(\lambda^{\prime}\right) \subset \operatorname{int} D_{i}^{\rho^{\prime}} .
$$

Since $\bigcup_{t>0} \mathcal{S}_{\leq t}$ is path connected and since the eigenvalues depend continuously on the entries of the matrix, there is $\delta>0$ such that there exist $t>0$ and $g \in$ int $_{\leq t}^{\rho}$ for $\rho=\rho^{\prime}-\delta$ with an eigenvalue $\lambda \in \operatorname{spec} g$ and $\frac{1}{t} \log |\lambda| \geq$ $\frac{1}{t^{\prime}} \log \left|\lambda^{\prime}\right|-\varepsilon$. Furthermore, by Theorem 5.2(ii) we may choose $\delta$ small enough such that $\mathbb{P} E(\lambda) \subset$ int $D_{i}^{\rho}$. Hence $\kappa_{i}(\rho) \geq \kappa_{i}\left(\rho^{\prime}\right)-2 \varepsilon$. By monotonicity this holds for all $\rho \geq \rho^{\prime}-\delta$.

Lower semicontinuity of inf $\Sigma_{F l}\left(D_{i}^{\rho}\right)$ is proved in the same way.

(ii) Monotonicity is again obvious, and upper semicontinuity follows from Theorem 3.11 of $[\mathrm{CKj}]$.

(iii) This follows from (i) and (ii) above and Theorem 4.5(iii).

(iv) By Theorem 3.1(ii) $\max \Sigma_{M o}\left(E_{i}^{\rho}\right)$ is attained in a Lyapunov exponent $\lambda(u, p)$ with $(u, p) \in \mathcal{E}_{i}^{\rho}$, hence $\pi_{\mathbb{P}} \omega(u, p) \subset E_{i}^{\rho} \subset \operatorname{int} D_{i}^{\rho^{\prime}}$ by (5.4). Thus, by Theorem 4.11, we have that $\lambda(u, p) \in c \ell \Sigma_{F l}\left(D_{i}^{\rho^{\prime}}\right)$. The same argument works for $\min \Sigma_{M o}\left(E_{i}^{\rho}\right)$, and the assertion follows.

5.5. Remark. Assertion (iii) can also be proved in a different way. By Theorem 4.5 (iii) the supremal Lyapunov exponent $\kappa(\rho)$ satisfies $\kappa(\rho)=\sup \Sigma_{L y}^{\rho}=\sup \Sigma_{F l}\left(D_{k}^{\rho}\right)$, thus it is the supremum over eigenvalues of all periodic matrix functions. Hence it coincides with the supremal Bohl exponent which describes uniform exponential growth behavior, cf. e.g. [DK], Chapter III. But upper semicontinuity of extremal Bohl exponents is a classical result, see e.g. [DK], Theorem III.4.6.

The following corollary shows in particular, that up to a 'thin' set of $\rho$-values the Floquet spectrum determines the Lyapunov spectrum. 
5.6. Corollary. Assume $\left(\mathrm{H}^{\rho}\right)$ and the $\rho-\rho^{\prime}$ inner pair condition (I). Then for each $i=1, \ldots, k$, the sets of continuity points of the two maps $\rho \mapsto \Sigma_{F l}\left(D_{i}^{\rho}\right)$ and $\rho \mapsto \Sigma_{M o}\left(E_{i}^{\rho}\right)$ agree. At each continuity point we have $c \ell \Sigma_{F l}\left(D_{i}^{\rho}\right)=\Sigma_{M o}\left(E_{i}^{\rho}\right)$, in particular, if $\rho$ is a continuity point for all $i=1, \ldots, k$, then

$$
\bigcup_{i=1}^{k} c \ell \Sigma_{F l}\left(D_{i}^{\rho}\right)=\Sigma_{L y}^{\rho}=\bigcup_{i=1}^{k} \Sigma_{M o}\left(E_{i}^{\rho}\right)=\Sigma_{M o}^{\rho} .
$$

Note that there are at most countably many points of discontinuity.

Proof. Let $\rho_{1}$ be a continuity point of $c \ell \Sigma_{F l}\left(D_{i}^{\rho}\right)=\left[\kappa_{i}^{*}(\rho), \kappa_{i}(\rho)\right]$. We have to show left continuity of $\rho \mapsto \Sigma_{M o}\left(E_{i}^{\rho}\right)$ at $\rho=\rho_{1}$ : For $\varepsilon>0$ let $\delta>0$ be such that $\kappa_{i}\left(\rho_{1}\right)-\varepsilon \leq \kappa_{i}(\rho) \leq \kappa_{i}\left(\rho_{1}\right)$ for all $\rho \in\left[\rho_{1}-\delta, \rho_{1}\right]$. Then, by Theorem 5.4 (iv), $\kappa_{i}\left(\rho_{1}\right)-\varepsilon \leq \max \Sigma_{M o}\left(E_{i}^{\rho}\right) \leq \kappa_{i}\left(\rho_{1}\right)$ for all $\rho \in\left(\rho_{1}-\delta, \rho_{1}\right)$. By assumption $\Sigma_{F l}\left(D_{i}^{\rho}\right)$ is right continuous at $\rho=\rho_{1}$. This implies that $\max \Sigma_{M o}\left(E_{i}^{\rho_{1}}\right) \leq \kappa_{i}\left(\rho_{1}\right)$. Similar arguments hold for $\kappa_{i}^{*}(\rho)$ and hence the assertion follows.

If $\rho_{1}>0$ is a continuity point of $\Sigma_{M o}\left(E_{i}^{\rho}\right)$, then $\max \Sigma_{M o}\left(E_{i}^{\rho_{1}}\right) \leq \kappa_{i}\left(\rho_{1}\right)$ and the proof follows from the fact that $c \ell \Sigma_{F l}\left(D_{i}^{\rho}\right) \subset \Sigma_{M o}\left(E_{i}^{\rho}\right)$ for all $\rho>0$.

These arguments show at the same time that at continuity points of the spectral intervals we have $c \ell \Sigma_{F l}\left(D_{i}^{\rho}\right)=\Sigma_{M o}\left(E_{i}^{\rho}\right)$.

Finally, the monotonicity of the considered maps implies that there are at most countably many points of discontinuity.

5.7. Remark. While the intervals of the Lyapunov spectrum can overlap (see Remark 4.7 (ii)), we have at the continuity points of the spectrum that the minima (and the maxima) of the spectral intervals are strictly separated, compare Remark 3.3 .

5.8. Remark. In the two dimensional case $d=2$, we know from Corollary 4.10 that $c \ell \Sigma_{F \ell}(\rho)=\Sigma_{L y}(\rho)=\Sigma_{M o}(\rho)$ for all $\rho \geq 0$. From the results above we obtain for the continuity of the spectral intervals: Let $I \subset[0, \infty)$ be an interval with $D^{\rho}=\mathbb{P}^{1}$ for all $\rho \in I$, then $\Sigma_{L y}(\rho)=c \ell \Sigma_{F \ell}\left(D^{\rho}\right)$ is continuous in $\rho$ for $\rho \in I$. This follows directly from Corollary 4.10 (iii) and Theorem 5.4 (iii). If, on the other hand, $I \subset[0, \infty)$ is an interval with two distinct chain control sets $E_{1}^{\rho}=c \ell D_{1}^{\rho}$ and $E_{2}^{\rho}=c \ell D_{2}^{\rho}$ for all $\rho \in I$, then the maps $\rho \mapsto c \ell \Sigma_{F \ell}\left(D_{i}^{\rho}\right)=\Sigma_{M o}\left(E_{i}^{\rho}\right)$ for $i=1,2$ are continuous in $\rho$ on $I$. This follows from the fact that in this case the entire spectrum consists of real parts of eigenvalues of constant matrices in $U^{\rho}$, see Corollary 4.10 (i). Hence the spectral intervals can only be discontinuous at a point $\rho$ with the property: there is exactly one chain control set $E^{\rho}=\mathbb{P}^{1}$, and two distinct main control sets $D_{1}^{\rho}$ and $D_{2}^{\rho}$, compare Theorem 5.2 (iv). Note that under the assumptions of this section, for each given $U \subset \mathbb{R}^{m}$ there can be at most one point $\rho$ with this property. At these $\rho$-points, the spectral intervals can, in fact, be discontinuous, as the discussion of the linear oscillator in Section 6 of [CKe] shows.

At the continuity points of the spectral intervals we obtain the following picture. The Lyapunov spectrum consists of the Floquet spectrum over the interior of the main control sets and at most $d$ additional points. Each such component of the Lyapunov spectrum is a compact interval (missing at most $d$ points in the interior). To each such interval is associated a unique Morse set of the projected flow $\mathbb{P} \Phi$, and hence a (constant dimensional) subbundle decomposition of $\mathcal{U} \times \mathbb{R}^{d}$. The order between the Morse sets reflects the order of the spectral intervals, but these intervals 
may overlap, even in interior points. The intervals of the Lyapunov spectrum inherit the upper semicontinuity property of the Morse spectrum with respect to perturbations (see [CKj], Corollary 3.12).

Furthermore, in $[\mathrm{CKj}]$, Section 5 results on the relation of the Morse spectrum to the dichotomy spectrum $\Sigma_{d i c}([\mathrm{SaS}])$, the topological spectrum $\Sigma_{t o p}([\mathrm{SaZ}])$, and the Oseledeč spectrum $\Sigma_{O s}$ are given using ergodic theory of the Morse spectrum. These results immediately apply to the Lyapunov spectrum of families of time varying matrices in the current set up. With respect to the Oseledec spectrum we mention the following additional properties. The Oseledeč spaces need not be contained in the closure of main control sets, as Example 4.9 shows. However, every point in the interior of a main control set 'is' an Oseledec space for some ergodic measure $\mu$ on $\mathcal{U}$, compare Theorem 4.1 (i). Hence every point in the interior of a chain control set 'is' an Oseledeč space in the situation of Theorem 5.1 (iv), i.e. for $\rho \neq \rho_{i}^{n}$. For each ergodic $\mu$ on $\mathcal{U}$ the Oseledec spectrum $\Sigma_{O s}(\mu)$ consists of $\lambda_{1}(\mu)<\cdots<\lambda_{s}(\mu)$ with $1 \leq s \leq d$. We have that $\ell \leq s$, where $\ell$ is the number of chain control sets, and simple examples show that $\ell<s$ is possible. Similarly, the decomposition of $\mathcal{U} \times \mathbb{R}^{d}$ into Oseledec spaces can be finer (with $\mu$-probability 1) than the decomposition according to the Morse spectrum. But note that the Oseledec decomposition is, in general, only measurable.

Since the shift on $\mathcal{U}$ is chain recurrent, we obtain from $[\mathrm{CKj}]$, formulae (5.13) and (5.17), the following chain of inclusions for the different spectral concepts applied to the family (2.1) of time varying matrices

$$
\begin{aligned}
& \Sigma_{F l} \subset \bigcup_{\mu \text { ergodic }} \Sigma_{O s}(\mu) \subset \Sigma_{L y} \subset \Sigma_{M o}=\Sigma_{t o p}=\Sigma_{d i c} \\
& \partial \Sigma_{M o}\left(E_{j}\right) \subset \Sigma_{L y} \text { for } j=1 \ldots \ell
\end{aligned}
$$

At the continuity points of the spectra all inclusions in (5.7) are equalities, with $\Sigma_{F l}$ replaced by its closure, $c \ell \Sigma_{F l}$.

\section{REFERENCES}

[AK] L. Arnold and W. Kliemann, Large deviations of linear stochastic differential equations, Stochastic Differential Systems, J. H. Engelbrecht and W. Schmidt (eds.) Spinger-Verlag (1987), 117-151.

[AKO] L. Arnold, W. Kliemann, and E. Oeljeklaus, Lyapunov exponents of linear stochastic systems, Lyapunov Exponents, L. Arnold and V. Wihstutz (eds.), Lecture notes in Math. 1186, Springer-Verlag (1986), 85-125. MR 87k:93095

[BSM] C.J.B. Barros and L.A.B. San Martin, On the number of control sets on projective spaces, Instituto de Matematica, RP 67/94, Universidade Estadual de Campinas, Campinas (Brasil).

[BVGN] B.F. Bylov, R.E. Vinograd, D.M. Grobman, and V.V. Nemytskii, Theory of Lyapunov Exponents, 1966, Moscow : Nauka (in Russian). MR 34:6234

[Ce] L. Cesari, Asymptotic Behavior and Stability Problems in Ordinary Differential Equations, 3rd ed., Springer-Verlag, 1971. MR 50:2582

[CKa] F. Colonius and W. Kliemann, Infinite time optimal control and periodicity, Appl. Math. Optim. 20 (1989), 113-130. MR 90c:49068

[CKb] F. Colonius and W. Kliemann, Stability radii and Lyapunov exponents, Control of Uncertain Systems, D. Hinrichsen and B. Martensson (eds.), Birkhäuser (1990), 19-55. MR 93i:93091

[CKc] F. Colonius and W. Kliemann, Remarks on ergodic theory of stochastic flows and control flows, Diffusion Processes and Related Problems in Analysis, Vol. II, M. Pinsky and V. Wihstutz (eds.), Birkhäuser, (1991), pp. 203-240. MR 93i:60110 
[CKd] F. Colonius and W. Kliemann, Stabilization of linear uncertain systems, Modeling, Estimation and Control of Systems with Uncertainty, G.B. DiMasi, A. Gombani, A.B. Kurzhansky (eds.) Birkhäuser (1991), 76-90. MR 92:03

[CKe] F. Colonius and W. Kliemann, Maximal and minimal Lyapunov exponents of bilinear control systems, J. Diff. Equations 101 (1993), 232-275. MR 93j:93096

[CKf] F. Colonius and W. Kliemann, Some aspects of control systems as dynamical systems, J. Dynamics Diff. Equations 5 (1993), 469-494. MR 94g:93063

[CKg] F. Colonius and W. Kliemann, Linear control semigroups acting on projective space, J. Dynamics Diff. Equa. 5 (1993), 495-528. MR 94g:93064

[CKh] F. Colonius and W. Kliemann, Limit behavior and genericity for nonlinear control systems, J. Differential Equations 109 (1994), 8-41. MR 94:11

[CKi] F. Colonius and W. Kliemann, Asymptotic null-controllability of bilinear systems, Geometry in Nonlinear Control and Differential Inclusions (Warsaw, 1993), Banach Center Publ., 32, Polish Acad. Sci., Warsaw. 1995, 139-148. CMP 96:05

[CKj] F. Colonius and W. Kliemann, The Morse spectrum of linear flows on vector bundles, Trans. Amer. Math. Soc. 348 (1996), 4355-4388.

[Co] C. Conley, Isolated Invariant Sets and the Morse Index, CBMS Regional Conference Series, no. 38, American Mathematics Society, Providence, R.I., 1978. MR 80c:58009

[DK] Ju.L. Daleckii and M. G. Krein, Stability of Solutions of Differential Equations in Banach Space, Translations of Math. Monographs Vol. 43, Amer. Math. Soc., Providence R.I., 1974. MR 50:5126

[Ha] W. Hahn, The Stability of Motion, Springer-Verlag, 1967. MR 36:6716

[Is] A. Isidori, Nonlinear Control Systems, Springer-Verlag, 2nd ed., 1989. MR 90m:93068

[JPS] R. A. Johnson, K.J. Palmer, and G.R. Sell, Ergodic properties of linear dynamical system, SIAM J. Math. Anal. 18 (1987), 1-33. MR 88a:58112

[Jo] E. C. Joseph, Stability radii of two dimensional bilinear systems: Lyapunov exponents approach, 1993, Ph.D. Thesis, Department of Mathematics, Iowa State University, Ames.

[Ly] M.A. Lyapunov, General Problems in the Stability of Motion, (in Russian) Comm. Soc. Math. Kharkow, 1892 (reprinted in Ann.Math.Studies 17, Princeton (1949)).

[Ma] R. Mañé, Ergodic Theory and Differentiable Dynamics, Springer-Verlag, 1987. MR 88c: 58040

[Mi] R.K. Miller, Almost periodic differential equations as dynamical systems with applications to the existence of almost periodic solutions, J. Diff. Equations 1 (1965), 337-345. MR 32:2690

[Os] V. I. Oseledeč, A multiplicative ergodic theorem. Lyapunov characteristic numbers for dynamical systems, Trans. Moscow Math. Soc. 19 (1968), 197-231. MR 39:1629

[SaS] R. J. Sacker and G.R. Sell, A spectral theory for linear differential systems, J. Diff. Equations 37 (1978), 320-358. MR 58:18604

[SaZ] D. Salamon and E. Zehnder, Flows on vector bundles and hyperbolic sets, Trans. Amer. Math. Soc. 306 (1988), 623-649. MR 89f:58112

[SMT] L.A.B. San Martin and P.A. Tonelli, Semigroup actions on homogeneous spaces, Semigroup Forum 50 (1995), 59-88.

[Sei] G. Seifert, Almost periodic solutions for almost periodic systems of ordinary differential equations, J. Diff. Equations 2 (1966), 305-319. MR 34:4613

[Sel] G. R. Sell, Nonautonomous differential equations and topological dynamics I, II, Trans. AMS 127 (1967), 241-262 and 263-283. MR 35:3187

Institut für Mathematik, Universität Augsburg, 86135 Augsburg, Germany

Department of Mathematics, Iowa State University, Ames, Iowa 50011 